\title{
Convolution identities for twisted Eisenstein series and twisted divisor functions
}

Daeyeoul Kim ${ }^{1}$ and Abdelmejid Bayad ${ }^{2 *}$

${ }^{\text {"Correspondence: }}$

abayad@maths.univ-evry.fr

²Département de mathématiques, Université d'Evry Val d'Essonne, Bâtiment I.B.G.B.I., 3ème étage, 23 Boulevard de France, 91037 Evry cedex, France

Full list of author information is available at the end of the article

\begin{abstract}
We are motivated by Ramanujan's recursion formula for sums of the product of two Eisenstein series (Berndt in Ramanujan's Notebook, Part II, 1989, Entry 14, p.332) and its proof, and also by Besge-Liouville's convolution identity for the ordinary divisor function $\sigma_{k-1}(n)$ (Williams in Number Theory in the Spirit of Liouville, vol. 76, 2011, Theorem 12.3). The objective of this paper is to introduce and prove convolution identities for the twisted divisor functions $\sigma_{k-1}^{*}(n)$ as well as for the twisted Eisenstein series $S_{2 k+2, \chi_{0}}$ and $S_{2 k+2, \chi_{1}}, S_{2 k+2,}^{*} S_{2 k+2, \chi_{0}}^{*}$ and $S_{2 k+2, \chi_{1}}^{*}$. As applications based on our main results, we establish many interesting identities for pyramidal, triangular, Mersenne, and perfect numbers. Moreover, we show how our main results can be used to obtain arithmetical formulas for the number of representations of an integer $n$ as the sums of $s$ squares.
\end{abstract}

\section{Introduction and statement of main results}

Throughout this paper, let $\mathfrak{H}=\{\tau \in \mathbb{C} \mid \operatorname{Im} \tau>0\}$ be the complex upper half-plane. Let $S_{k}$ denote the normalized Eisenstein series of weight $k$ defined on $\mathfrak{H}$ by

$$
S_{k}:=S_{k}(\tau)=-\frac{B_{k}}{2 k}+\sum_{n=1}^{\infty} \sigma_{k-1}(n) q^{n}
$$

where $\sigma_{k-1}(n)=\sum_{d \mid n} d^{k-1}, q=e^{2 \pi i \tau}$, and $B_{k}$ is the $k$ th Bernoulli number.

Let $S_{k}^{*}, S_{k, \chi_{0}}^{*}$, and $S_{k, \chi_{1}}^{*}$ be the twisted Eisenstein series defined by

$$
\begin{aligned}
S_{k}^{*} & :=S_{k}^{*}(\tau)=S_{k}(\tau)-S_{k}(2 \tau) \\
& =\sum_{n=1}^{\infty}\left(\sigma_{k-1}(n)-\sigma_{k-1}\left(\frac{n}{2}\right)\right) q^{n}=\sum_{n=1}^{\infty} \sigma_{k-1}^{*}(n) q^{n} .
\end{aligned}
$$

For $i=0,1$, we define the twisted Eisenstein series by

$$
S_{k, \chi_{i}}(\tau):=\sum_{n=1}^{\infty} \chi_{i}(n) \sigma_{k-1}(n), \quad S_{k, \chi_{i}}^{*}(\tau):=\sum_{n=1}^{\infty} \chi_{i}(n) \sigma_{k-1}^{*}(n) q^{n}
$$

where $\sigma_{k-1}^{*}(n)$ is the twisted divisor function given by

$$
\sigma_{k-1}^{*}(n)=\sum_{d \mid n, \frac{n}{d} \text { odd }} d^{k-1}
$$

C 2013 Kim and Bayad; licensee Springer. This is an Open Access article distributed under the terms of the Creative Commons Attribution License (http://creativecommons.org/licenses/by/2.0), which permits unrestricted use, distribution, and reproduction in any medium, provided the original work is properly cited. 


$$
\chi_{1}(n)=\left\{\begin{array}{ll}
1, & \text { if } n \text { is odd, } \\
0, & \text { otherwise }
\end{array} \text { and } \quad \chi_{0}(n)= \begin{cases}1, & \text { if } n \text { is even } \\
0, & \text { otherwise }\end{cases}\right.
$$

Consider the differential operator $D=\frac{1}{2 \pi i} \frac{d}{d \tau}=q \frac{d}{d q}$ such that

$$
D\left(\sum_{n=1}^{\infty} a_{n} q^{n}\right)=\sum_{n=1}^{\infty} n a_{n} q^{n}
$$

We are motivated by Ramanujan's recursion formula for $S_{2 n+2}$ [1, Entry 14, p.332] and its proof, and also by Besge-Liouville's convolution identity for the divisor function $\sigma_{k-1}(n)$ [2, Theorem 12.3]. Inspired by these identities, we prove new identities for the convolution sums of various divisor functions $\sigma_{k-1}(n), \sigma_{k-1}^{*}(n)$ as well as recursion formulas for the twisted Eisenstein series $S_{2 k+2}^{*}, S_{2 k+2, \chi_{0}}^{*}$, and $S_{2 k+2, \chi_{1}}^{*}$. Several arithmetical applications based on our results are given.

The problem of convolution sums of the divisor function $\sigma_{1}(n)$ and the theory of Eisenstein series has recently attracted considerable interest with the emergence of quasimodular tools. For further details, we can refer to the works of Royer [3], Ramakrishnan and Sahu [4], and Alaca and Williams [5, 6], and the references therein. In connection with the classical Jacobi theta and Euler functions, other aspects of the function $\sigma_{1}(n)$ are explored by Adiga in [7] and by Simsek in [8]. In this paper, we prove the following results. First, we state the most important identities for the convolution sums of divisor functions as follows.

Theorem 1.1 Let $k \in \mathbb{N}$ and $N \in \mathbb{N}$, where $k, N \geq 2$. We have the identities in Table 1 .

Our next results follow from the identities in Theorem 1.1. Thus, we obtain the following identities for the Eisenstein series.

Theorem 1.2 Let $k \in \mathbb{N}$ and $n \in \mathbb{N}$, where $k, n \geq 2$. We have the identities in Table 2.

Let $p$ be a prime number, and let $l$ be any positive integer. We introduce the twisted divisor function

$$
\sigma_{s}^{\prime \prime}\left(N ; p^{l}\right):=\sum_{d \mid N, \frac{N}{d} \neq 0} d_{\left(\bmod p^{l}\right)}
$$

Table 1 Identities of divisor functions

\begin{tabular}{ll}
\hline Identities of convolution sums & Reference \\
\hline$\sum_{s=0}^{k-1}\left(\begin{array}{c}2 k \\
2 s+1\end{array}\right)\left(\sum_{m=1}^{N-1} \sigma_{2 k-2 s-1}(m) \sigma_{2 s+1}(N-m)\right)$ & {$[2$, Theorem 12.3] } \\
$\quad=\frac{2 k+3}{4 k+2} \sigma_{2 k+1}(N)+\left(\begin{array}{l}k \\
6\end{array}-N\right) \sigma_{2 k-1}(N)$ & \\
$\quad+\frac{1}{2 k+1} \sum_{j=2}^{k}\left(\begin{array}{c}2 k+1 \\
2 j\end{array}\right) B_{2 j} \sigma_{2 k+1-2 j}(N)$ & \\
$\sum_{s=0}^{k-1}\left(\begin{array}{c}2 k \\
2 s+1\end{array}\right)\left(\sum_{m=1}^{N-1} \sigma_{2 k-2 s-1}^{*}(m) \sigma_{2 s+1}^{*}(N-m)\right)$ & \\
$\quad=\frac{1}{2}\left\{\sigma_{2 k+1}^{*}(N)-N \sigma_{2 k-1}^{*}(N)\right\}$ & \\
$\sum_{s=0}^{k-1}\left(\begin{array}{c}2 k+1 \\
2 s+1\end{array}\right)\left(\sum_{m=1}^{N} \sigma_{2 k-2 s-1}(2 m-1) \sigma_{2 s+1}(2 N-2 m+1)\right)$ \\
$\quad=\frac{1}{4} \sigma_{2 k+1}^{*}(2 N)$ \\
$\sum_{s=0}^{k-1}\left(\begin{array}{l}2 k \\
2 s+1\end{array}\right) \sum_{m=1}^{2 N-1}(-1)^{m+1} \sigma_{2 k-2 s-1}^{*}(m) \sigma_{2 s+1}^{*}(2 N-m)$ \\
$\quad=\frac{1}{2}\left(2 N \cdot \sigma_{2 k-1}^{*}(2 N)\right)$ \\
\hline
\end{tabular}


Table 2 Identities of divisor functions

\begin{tabular}{|c|c|}
\hline Identities of Eisenstein series & Reference \\
\hline $\begin{aligned}-\frac{(n+2)(n+3)}{2 n(n-1)} S_{n+2}= & -20\left(\begin{array}{c}n-2 \\
2\end{array}\right) S_{4} S_{n-2} \\
& +\sum_{r=1}^{[(n-2) / 4]}\left(\begin{array}{c}n-2 \\
2 r\end{array}\right)\{(n+3-5 r)(n-8-5 r) \\
& -5(r-2)(r+3)\} S_{2 r+2} S_{n-2 r}\end{aligned}$ & {$[1$, Entry 14, p. 332] } \\
\hline $\begin{aligned} S_{2 n+2}^{*}(\tau)= & D\left(S_{2 n}^{*}(\tau)\right) \\
& +2 \sum_{s=0}^{n-1}\left(\begin{array}{c}2 n \\
2 s+1\end{array}\right) S_{2 n-2 s}^{*}(\tau) S_{2 s+2}^{*}(\tau)\end{aligned}$ & Theorem 3.2 \\
\hline $\begin{aligned} S_{2 k+2, \chi_{0}}^{*}(\tau) & =4 \sum_{s=0}^{k-1}\left(\begin{array}{c}2 k \\
2 s+1\end{array}\right) S_{2 k-2 s, \chi_{1}}^{*}(\tau) S_{2 s+2, \chi_{1}}^{*}(\tau) \\
& =4 \sum_{s=0}^{k-1}\left(\begin{array}{c}2 k \\
2 s+1\end{array}\right) S_{2 k-2 s, \chi_{1}}(\tau) S_{2 s+2, \chi_{1}}(\tau)\end{aligned}$ & Lemma 3.3 \\
\hline $\begin{aligned} D\left(S_{2 k, \chi_{0}}^{*}(\tau)\right)= & 2 \sum_{s=0}^{k-1}\left(\begin{array}{c}2 k \\
2 s+1\end{array}\right) \\
& \times\left(S_{2 k-2 s, \chi_{1}}^{*}(\tau)-S_{2 k-2 s, \chi_{0}}^{*}(\tau)\right) S_{2 s+2}^{*}(\tau)\end{aligned}$ & Lemma 3.3 \\
\hline
\end{tabular}

We define

$$
\left[\begin{array}{c}
n \\
m
\end{array}\right]_{\{p, l\}}:=\frac{\left(\frac{p^{l n}-1}{p^{n}-1}\right)}{\left(\frac{p^{l m}-1}{p^{m}-1}\right)\left(\frac{p^{l(n-m)}-1}{p^{n-m}-1}\right)}\left(\begin{array}{l}
n \\
m
\end{array}\right)
$$

In Section 3.2 , we establish the following less definitive result.

Theorem 1.3 Let $k \geq 1$ and $N, l \geq 2$.

$$
\begin{aligned}
\sum_{s=0}^{k-1}\left[\begin{array}{c}
2 k \\
2 s+1
\end{array}\right] \sum_{\{2, l\}}^{N-1} \sigma_{m=1}^{*} \sigma_{k-2 s-1}\left(2^{l} m ; 2^{l}\right) \sigma_{2 s+1}^{*}\left(2^{l}(N-m) ; 2^{l}\right) \\
=\frac{1}{2}\left(\frac{1}{2^{l}} \sigma_{2 k+1}^{*}\left(2^{l} N ; 2^{l}\right)-2^{l} N \sigma_{2 k-1}^{*}\left(2^{l} N ; 2^{l}\right)\right. \\
\left.+\sum_{i=1}^{l-1} \frac{1}{2^{i+1}} \sigma_{2 k+1}^{*}\left(2^{i} N ; 2^{i}\right)+N \sum_{i=1}^{l-1} 2^{i} \sigma_{2 k-1}^{*}\left(2^{i} N ; 2^{i}\right)\right) .
\end{aligned}
$$

Finally, in the last Section 4, we show how to apply our main results to study some questions related to the famous problem

$$
\sigma_{1}(n)=\sigma_{1}(n+1)
$$

and its variants. Moreover, we consider similar questions on the arithmetic function $\sigma_{1}^{*}(n)$. Many remarks and results are established.

\section{The $p^{s}$ th scalar multiplicative function}

Let $p$ be a prime number, and let $i$ be a nonnegative integer. A function $f: \mathbb{N} \rightarrow \mathbb{C}$ is called a $p^{i}$ th scalar function if $f(p x)=p^{i} f(x)$ for all integers $x$.

Moreover, a $p^{i}$ th scalar function $f(x)$ is called a $p^{i}$ th scalar multiplicative function if and only if $f(x y)=f(x) f(y)$ for all positive integers $x, y$ such that $(x, y)=1$.

\section{Example 2.1}

1. Let $f(x)=x$. Then $f(x)$ is a $p$ th scalar multiplicative function.

2 . Let $s$ be a nonnegative integer; we recall that

$$
\sigma_{s}^{*}(N ; p)=\sum_{d \mid N, \frac{N}{d} \neq 00} d_{(\bmod p)}^{s}, \quad \text { and } \quad \sigma_{s}^{*}(N)=\sigma_{s}^{*}(N ; 2)
$$


and we define the function

$$
\sigma_{s, r}(N ; m)=\sum_{d \mid N, d \equiv r} d_{(\bmod m)}
$$

Then the function $\bar{\sigma}_{s}(N ; p):=\sum_{r=1}^{p-1} \sigma_{s, r}(N ; p)$ is a $p^{0}$ th scalar multiplicative function.

3. The function $\sigma_{1,1}(N ; 2)$ is a $2^{0}$ th scalar multiplicative function.

Theorem 2.2 The divisor function $\sigma_{s}^{\prime \prime}(N ; p)$ is a $p^{s}$ th scalar multiplicative function.

Proof Let $r l=p^{m} N$ and $l \neq 0(\bmod p)$ for some $r, l \in \mathbb{N}$ and prime $p$. Since $l$ does not divide $p$, we can write $r=p^{m} d$ for some $d \in \mathbb{N}$. Therefore, we have

$$
\begin{aligned}
\sigma_{s}^{* \prime}\left(p^{m} N ; p\right) & =\sum_{r \mid p^{m} N \frac{p^{m} N}{r} \neq 00} r^{s}=\sum_{d \mid N \frac{N}{d} \neq 0}\left(p^{m} d\right)^{s}(\bmod p) \\
& =p^{m s} \sum_{\substack{d \mid N \\
\frac{N}{d} \neq 0}} d^{s}=p^{m s} \sigma_{s}^{*}(N ; p) .
\end{aligned}
$$

We claim that $\sigma_{s}^{*}(M N ; p)=\sigma_{s}^{*}(M ; p) \sigma_{s}^{*}(N ; p)$, where $(M, N)=1$. If $p \nmid M N$, then

$$
\sigma_{s}^{*}(M N ; p)=\sigma_{s}(M N)=\sigma_{s}(M) \sigma_{s}(N)=\sigma_{s}^{*}(M ; p) \sigma_{s}^{*}(N ; p) .
$$

Assume that $p \mid M$ and $p \nmid N$ and $M=p^{l} M^{\prime}$. Hence, we have

$$
\begin{aligned}
\sigma_{s}^{*}(M N ; p) & =\sigma_{s}^{*}\left(p^{l} M^{\prime} N ; p\right)=p^{l} \sigma_{s}^{*}\left(M^{\prime} N ; p\right) \\
& =p^{l} \sigma_{s}^{*}\left(M^{\prime} ; p\right) \sigma_{s}^{*}(N ; p)=\sigma_{s}^{*}\left(p^{l} M^{\prime} ; p\right) \sigma_{s}^{*}(N ; p) .
\end{aligned}
$$

Therefore $\sigma_{s}^{*}(M N ; p)=\sigma_{s}^{*}(M ; p) \sigma_{s}^{*}(N ; p)$. Then we get the theorem.

Proposition 2.3 Let $N \in \mathbb{N}$. For nonnegative integers $m$ and $n$, we have

$$
\sum_{k=1}^{N-1} \sigma_{i}^{*}\left(p^{m} k ; p^{m}\right) \sigma_{j}^{*}\left(p^{n}(N-k) ; p^{n}\right)=p^{i+j} \frac{\left(p^{i m}-1\right)\left(p^{j n}-1\right)}{\left(p^{i}-1\right)\left(p^{j}-1\right)} \sum_{k=1}^{N-1} \sigma_{i}^{*}(k ; p) \sigma_{j}^{*}(N-k ; p) .
$$

Proof We can see that

$$
\begin{aligned}
& \sum_{k=1}^{N-1} \sigma_{i}^{*}\left(p^{m} k ; p^{m}\right) \sigma_{j}^{*}\left(p^{n}(N-k) ; p^{n}\right) \\
& \quad=\sum_{k=1}^{N-1}\left\{\sigma_{i}\left(p^{m} k\right)-\sigma_{i}(k)\right\}\left\{\sigma_{j}\left(p^{n}(N-k)\right)-\sigma_{j}(N-k)\right\} .
\end{aligned}
$$


Then, by Theorem 2.2, we have

$$
\begin{aligned}
\sigma_{i}\left(p^{m} k\right)-\sigma_{i}(k)= & \left(\sigma_{i}\left(p^{m} k\right)-\sigma_{i}\left(p^{m-1} k\right)\right)+\left(\sigma_{i}\left(p^{m-1} k\right)-\sigma_{i}\left(p^{m-2} k\right)\right) \\
& +\left(\sigma_{i}\left(p^{m-2} k\right)-\sigma_{i}\left(p^{m-3} k\right)\right)+\cdots \\
& +\left(\sigma_{i}\left(p^{2} k\right)-\sigma_{i}(p k)\right)+\left(\sigma_{i}(p k)-\sigma_{i}(k)\right) \\
= & \sigma_{i}^{*}\left(p^{m} k ; p\right)+\sigma_{i}^{*}\left(p^{m-1} k ; p\right)+\sigma_{i}^{*}\left(p^{m-2} k ; p\right)+\cdots \\
& +\sigma_{i}^{*}\left(p^{2} k ; p\right)+\sigma_{i}^{*}(p k ; p) \\
= & \left(p^{m i}+p^{(m-1) i}+p^{(m-2) i}+\cdots+p^{2 i}+p^{i}\right) \sigma_{i}^{*}(k ; p) \\
= & p^{i} \frac{p^{m i}-1}{p^{i}-1} \sigma_{i}^{*}(k ; p) .
\end{aligned}
$$

Thus, using (8), we can write (7) as

$$
\sum_{k=1}^{N-1} \sigma_{i}^{*}\left(p^{m} k ; p^{m}\right) \sigma_{j}^{*}\left(p^{n}(N-k) ; p^{n}\right)=\sum_{k=1}^{N-1}\left\{p^{i} \frac{p^{m i}-1}{p^{i}-1} \sigma_{i}^{*}(k ; p)\right\}\left\{p^{j} \frac{p^{n j}-1}{p^{j}-1} \sigma_{j}^{*}(N-k ; p)\right\} .
$$

\section{Convolution relations on twisted Eisenstein series and their applications}

\subsection{Convolution relations on the twisted Eisenstein series $S_{2 n+2}^{*}(\tau)$}

We quote the following lemma from [2, Chap. 10, p.113].

Lemma 3.1 Let $f: \mathbb{Z} \rightarrow \mathbb{C}$ be an even function. Let $n \in \mathbb{N}$. Then

$$
\begin{gathered}
\sum_{\substack{(a, b, x, y) \in \mathbb{N}^{4} a x+b y=n x, y \text { odd } \\
=}}(f(a-b)-f(a+b)) \\
=f(0) \sigma_{1}(n / 2)+\sum_{\substack{d \in \mathbb{N} \\
d \mid n}}\left(\frac{n}{d}-d\right) f(d)-\sum_{\substack{d \in \mathbb{N} \\
d \mid n / 2}}\left(\frac{n}{d}-d\right) f(d) .
\end{gathered}
$$

Using the above lemma, we can prove our next result.

Theorem 3.2 Let $n>1$. Then

$$
S_{2 n+2}^{*}(\tau)=D\left(S_{2 n}^{*}(\tau)\right)+2 \sum_{s=0}^{n-1}\left(\begin{array}{c}
2 n \\
2 s+1
\end{array}\right) S_{2 n-2 s}^{*}(\tau) S_{2 s+2}^{*}(\tau) \text {. }
$$

Proof We take $f(x)=x^{2 k}$ in Lemma 3.1. The left-hand side of Lemma 3.1 is

$$
\begin{aligned}
& \sum_{\substack{(a, x, b, y) \in \mathbb{N}^{4} \\
a x+b y=n \\
x, y \text { odd }}}\left((a-b)^{2 k}-(a+b)^{2 k}\right) \\
& =\sum_{\substack{(a, x, b, y) \in \mathbb{N}^{4} \\
a x+b y=n \\
x, y \text { odd }}}\left(\sum_{r=0}^{2 k}\left(\begin{array}{c}
2 k \\
r
\end{array}\right)(-1)^{r} a^{2 k-r} b^{r}-\sum_{r=0}^{2 k}\left(\begin{array}{c}
2 k \\
r
\end{array}\right) a^{2 k-r} b^{r}\right)
\end{aligned}
$$




$$
\begin{aligned}
& =-2 \sum_{\substack{(a, x, b, y) \in \mathbb{N}^{4} \\
a x+b y=n \\
x, y \text { odd }}} \sum_{\substack{r=0 \\
r \text { odd }}}^{2 k}\left(\begin{array}{c}
2 k \\
r
\end{array}\right) a^{2 k-r} b^{r} \\
& =-2 \sum_{s=0}^{k-1}\left(\begin{array}{c}
2 k \\
2 s+1
\end{array}\right) \sum_{\substack{(a, x, b, y) \in \mathbb{N}^{4} \\
a x+b y=n \\
x, y \text { odd }}} a^{2 k-2 s-1} b^{2 s+1} \\
& =-2 \sum_{s=0}^{k-1}\left(\begin{array}{c}
2 k \\
2 s+1
\end{array}\right) \sum_{m=1}^{n-1} \sigma_{2 k-2 s-1}^{*}(m) \sigma_{2 s+1}^{*}(n-m) \text {. }
\end{aligned}
$$

Therefore, we deduce that

$$
\sum_{s=0}^{k-1}\left(\begin{array}{c}
2 k \\
2 s+1
\end{array}\right)\left(\sum_{m=1}^{N-1} \sigma_{2 k-2 s-1}^{*}(m) \sigma_{2 s+1}^{*}(N-m)\right)=\frac{1}{2}\left\{\sigma_{2 k+1}^{*}(N)-N \sigma_{2 k-1}^{*}(N)\right\} .
$$

From (10) and (2), we get the theorem.

\subsection{Proof of Theorem 1.3}

From (4) and (10), we obtain

$$
\begin{aligned}
& \sum_{s=0}^{k-1}\left[\begin{array}{c}
2 k \\
2 s+1
\end{array}\right]_{\{2, l\}} \sum_{m=1}^{N-1} \sigma_{2 k-2 s-1}^{*}\left(2^{l} m ; 2^{l}\right) \sigma_{2 s+1}^{*}\left(2^{l}(N-m) ; 2^{l}\right) \\
&=\sum_{s=0}^{k-1} \frac{2^{2 k}\left(2^{2 l k}-1\right)}{2^{2 k}-1} \sum_{s=0}^{k-1}\left(\begin{array}{c}
2 k \\
2 s+1
\end{array}\right) \sum_{m=1}^{N-1} \sigma_{2 k-2 s-1}^{*}(m) \sigma_{2 s+1}^{*}(N-m) \\
&=\sum_{s=0}^{k-1} \frac{2^{2 k}\left(2^{2 l k}-1\right)}{2^{2 k}-1} \cdot \frac{1}{2}\left\{\sigma_{2 k+1}^{*}(N)-N \sigma_{2 k-1}^{*}(N)\right\} \\
&=\frac{1}{2}\left[\left\{2^{2 k l}+2^{2 k(l-1)}+\cdots+2^{2(2 k)}+2^{2 k}\right\} \sigma_{2 k+1}^{*}(N)\right. \\
&\left.-N\left\{2^{2 k l}+2^{2 k(l-1)}+\cdots+2^{2(2 k)}+2^{2 k}\right\} \sigma_{2 k-1}^{*}(N)\right] .
\end{aligned}
$$

Then, the first term on the right-hand side of (11) can be written as

$$
\begin{aligned}
\left\{2^{2 k l}+2^{2 k(l-1)}+\cdots+2^{2(2 k)}+2^{2 k}\right\} \sigma_{2 k+1}^{*}(N) & \left\{\frac{\left(2^{l}\right)^{2 k+1}}{2^{l}}+\frac{\left(2^{l-1}\right)^{2 k+1}}{2^{l-1}}+\cdots+\frac{\left(2^{2}\right)^{2 k+1}}{2^{2}}+\frac{2^{2 k+1}}{2}\right\} \sigma_{2 k+1}^{*}(N) \\
= & \frac{1}{2^{l}} \sigma_{2 k+1}^{*}\left(2^{l} N\right)+\frac{1}{2^{l-1}} \sigma_{2 k+1}^{*}\left(2^{l-1} N\right)+\cdots+\frac{1}{2^{2}} \sigma_{2 k+1}^{*}\left(2^{2} N\right)+\frac{1}{2} \sigma_{2 k+1}^{*}(2 N) \\
= & \left(\frac{1}{2^{l}} \sigma_{2 k+1}\left(2^{l} N\right)-\frac{1}{2^{l}} \sigma_{2 k+1}\left(2^{l-1} N\right)\right)+\left(\frac{1}{2^{l-1}} \sigma_{2 k+1}\left(2^{l-1} N\right)-\frac{1}{2^{l-1}} \sigma_{2 k+1}\left(2^{l-2} N\right)\right) \\
& +\cdots+\left(\frac{1}{2^{2}} \sigma_{2 k+1}\left(2^{2} N\right)-\frac{1}{2^{2}} \sigma_{2 k+1}(2 N)\right)+\left(\frac{1}{2} \sigma_{2 k+1}(2 N)-\frac{1}{2} \sigma_{2 k+1}(N)\right) \\
= & \frac{1}{2^{l}}\left(\sigma_{2 k+1}\left(2^{l} N\right)-\sigma_{2 k+1}(N)\right)+\frac{1}{2^{l}}\left(\sigma_{2 k+1}\left(2^{l-1} N\right)-\sigma_{2 k+1}(N)\right)+\cdots
\end{aligned}
$$




$$
\begin{aligned}
& +\frac{1}{2^{2}}\left(\sigma_{2 k+1}(2 N)-\sigma_{2 k+1}(N)\right) \\
= & \frac{1}{2^{l}} \sigma_{2 k+1}^{*}\left(2^{l} N ; 2^{l}\right)+\frac{1}{2^{l}} \sigma_{2 k+1}^{*}\left(2^{l-1} N ; 2^{l-1}\right)+\frac{1}{2^{l-1}} \sigma_{2 k+1}^{*}\left(2^{l-2} N ; 2^{l-2}\right) \\
& +\cdots+\frac{1}{2^{2}} \sigma_{2 k+1}^{*}(2 N ; 2) .
\end{aligned}
$$

Similarly, the second term on the right-hand side of (11) is

$$
\begin{aligned}
- & N\left\{2^{2 k l}+2^{2 k(l-1)}+\cdots+2^{2(2 k)}+2^{2 k}\right\} \sigma_{2 k-1}^{\prime \prime}(N) \\
& =-N\left\{2^{l} \sigma_{2 k-1}^{*}\left(2^{l} N ; 2^{l}\right)-2^{l-1} \sigma_{2 k-1}^{*}\left(2^{l-1} N ; 2^{l-1}\right)-\cdots-2 \sigma_{2 k-1}^{\prime \prime}(2 N ; 2)\right\} .
\end{aligned}
$$

Therefore, the proof is completed.

\subsection{Pyramidal numbers}

Let $\alpha$ be a fixed integer with $\alpha \geq 3$, and let

$$
\operatorname{Pyr}_{\alpha}(x)=\frac{1}{6} x(x+1)((\alpha-2) x+5-\alpha)
$$

denote the $\alpha$ th order pyramid number [9]. These combinatorial numbers play an important role in number theory and discrete mathematics. Using (10) with $k=1$, we derive

$$
\sum_{m=1}^{N-1} \sigma_{1}^{*}(m) \sigma_{1}^{*}(N-m)=\frac{1}{4}\left\{\sigma_{3}^{*}(N)-N \sigma_{1}^{*}(N)\right\}
$$

\begin{tabular}{|c|c|c|c|}
\hline$\sum_{k=1}^{N-1} \sigma_{1}^{*}(2 k) \sigma_{1}^{*}(2(N-k))$ & $\sum_{k=1}^{N-1} \sigma_{1}^{*}(4 k ; 4) \sigma_{1}^{*}(4(N-k) ; 4)$ & $\ldots$ & $\sum_{k=1}^{N-1} \sigma_{1}^{*}\left(2^{m} k ; 2^{m}\right) \sigma_{1}^{*}\left(2^{m}(N-k) ; 2^{m}\right)$ \\
\hline$(2-1)^{2}\left(\sigma_{3}^{*}(N)-N \sigma_{1}^{*}(N)\right)$ & $(4-1)^{2}\left(\sigma_{3}^{*}(N)-N \sigma_{1}^{*}(N)\right)$ & $\cdots$ & $\left(2^{m}-1\right)^{2}\left(\sigma_{3}^{*}(N)-N \sigma_{1}^{*}(N)\right)$ \\
\hline
\end{tabular}

Proposition 2.3 is a very efficient formula for the computation of the sum

$$
\sum_{k=1}^{N-1} \sigma_{i}^{*}\left(p^{m} k ; p^{m}\right) \sigma_{j}^{*}\left(p^{n}(N-k) ; p^{n}\right)
$$

For example, using Proposition 2.3 and (12), we get the interesting formulas in Table 3.

1. The pyramidal numbers $P_{5, x}$ are closely connected to the convolution sums

$$
\sum_{m=1}^{N-1} \sigma_{1}^{*}(m) \sigma_{1}^{\prime \prime}(N-m)
$$

Table 3 Values of $\sum_{k=1}^{N-1} \sigma_{1}^{*}\left(2^{m} k ; 2^{m}\right) \sigma_{1}^{*}\left(2^{m}(N-k) ; 2^{m}\right)$

Table 4 Examples for $P_{5, x}$ and $P_{5, x}^{*}$

\begin{tabular}{llllllllllllll}
\hline $\boldsymbol{x}$ & $\mathbf{2}$ & $\mathbf{3}$ & $\mathbf{4}$ & $\mathbf{5}$ & $\mathbf{6}$ & $\mathbf{7}$ & $\mathbf{8}$ & $\mathbf{9}$ & $\mathbf{1 0}$ & $\mathbf{1 1}$ & $\mathbf{1 2}$ & $\mathbf{1 3}$ & $\mathbf{1 4}$ \\
\hline$P_{5, x}$ & 6 & 18 & 40 & 75 & 126 & 196 & 288 & 405 & 550 & 726 & 936 & 1,183 & 1,470 \\
$P_{5, x}^{*}$ & 6 & 18 & 40 & 75 & 126 & 198 & 288 & 405 & 560 & 726 & 936 & 1,210 & 1,470 \\
\hline
\end{tabular}


In fact, if $N=2 q+1$ is a prime number, then from (12), we obtain

$$
\frac{1}{2} \sum_{m=1}^{q} \sigma_{1}^{*}(m) \sigma_{1}^{*}(2 q+1-m)=\frac{1}{2} q^{2}(q+1) .
$$

From Theorem 2.2, we deduce that

$$
\sum_{m=1}^{q} \hat{\sigma}_{1}^{*}(m) \hat{\sigma}_{1}^{*}(2 q+1-m)=\frac{1}{2} \sum_{m=1}^{q} \sigma_{1}^{*}(m) \sigma_{1}^{*}(2 q+1-m)=\operatorname{Pyr}_{5}(q),
$$

where

$$
\hat{\sigma}_{1}^{*}(t)= \begin{cases}\sigma_{1}^{*}(t) & \text { if } t \text { is odd } \\ \sigma_{1}^{*}\left(\frac{t}{2}\right) & \text { otherwise }\end{cases}
$$

In Table 4, we list the first thirteen values of

$$
P_{5, x}:=\frac{1}{2} x^{2}(x+1) \quad \text { and } \quad P_{5, x}^{*}:=\sum_{m=1}^{x} \hat{\sigma}_{1}^{*}(m) \hat{\sigma}_{1}^{*}(2 x+1-m) .
$$

According to Table 4 and Figure 1, we observe that if $2 x+1$ is prime, then $P_{5, x}$ coincides with $P_{5, x}^{*}$.

2. We consider the pyramidal numbers

$$
P_{3, x}:=\operatorname{Pyr}_{3}(x-1)=\frac{1}{6} x(x-1)(x+1)
$$

and the convolution sums

$$
P_{3, x}^{*}:=\sum_{x \geq 2 k} \sigma_{1}(k) \sigma_{1}(2 x+1-4 k) .
$$

From (21) (with $N=2 q+1$ prime), we obtain

$$
P_{3, q}=P_{3, q}^{*}=\operatorname{Pyr}_{3}(q-1)
$$
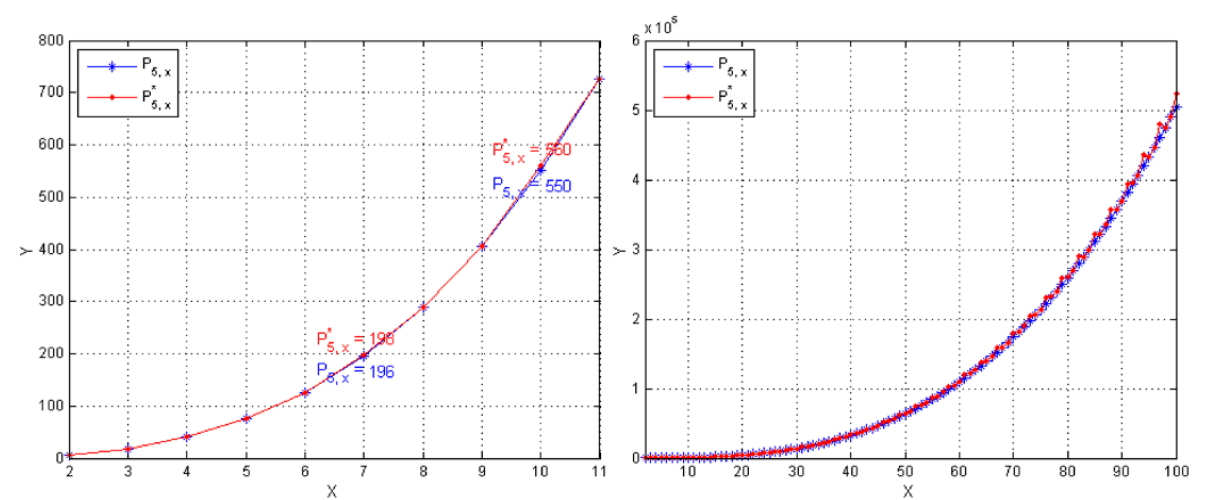

Figure $1 P_{5, x}$ and $P_{5, x}^{*}$. 
Table 5 Examples for $P_{3, x}$ and $P_{3, x}^{*}$

\begin{tabular}{llllllllllllll}
\hline $\boldsymbol{x}$ & $\mathbf{2}$ & $\mathbf{3}$ & $\mathbf{4}$ & $\mathbf{5}$ & $\mathbf{6}$ & $\mathbf{7}$ & $\mathbf{8}$ & $\mathbf{9}$ & $\mathbf{1 0}$ & $\mathbf{1 1}$ & $\mathbf{1 2}$ & $\mathbf{1 3}$ & $\mathbf{1 4}$ \\
\hline$P_{3, x}$ & 1 & 4 & 10 & 20 & 35 & 56 & 84 & 120 & 165 & 220 & 286 & 364 & 455 \\
$P_{3, x}^{*}$ & 1 & 4 & 9 & 20 & 35 & 52 & 84 & 120 & 160 & 220 & 281 & 360 & 455 \\
\hline
\end{tabular}
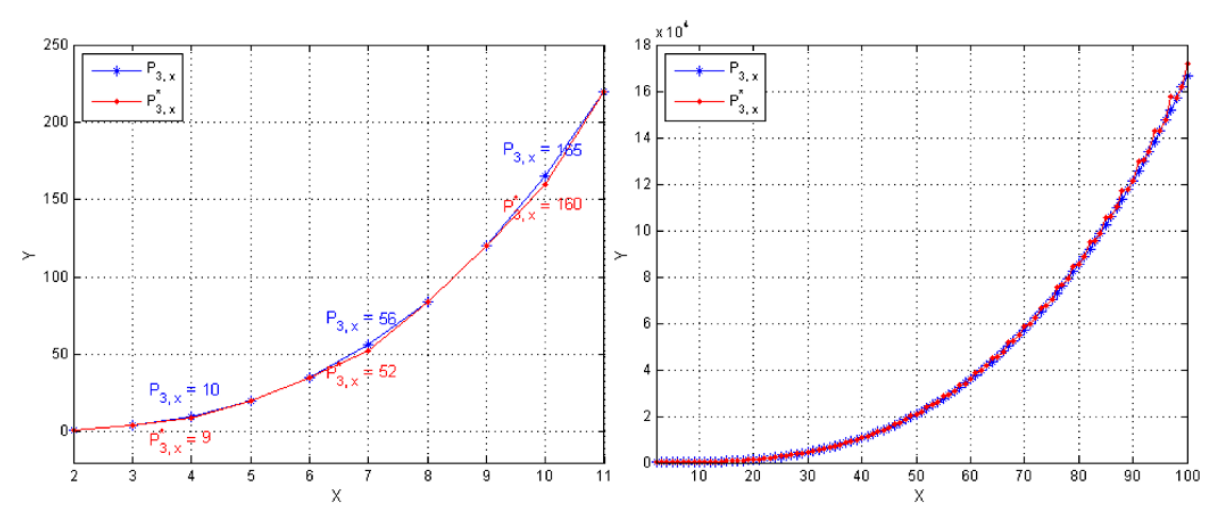

Figure $2 P_{3, x}$ and $P_{3, x}^{*}$.

We list the first thirteen values of $P_{3, x}$ and $P_{3, x}^{*}$ in Table 5. According to Table 5 and Figure 2 , we observe that if $2 x+1$ is prime, then $P_{3, x}$ coincides with $P_{3, x}^{*}$. We note that

$$
E: y^{2}=P_{3, x}=\frac{1}{6} x(x-1)(x+1)
$$

is an elliptic curve with $P=(2,1)=\left(2, \sqrt{P_{3,2}^{*}}\right) \in E(\mathbb{Q}) \backslash E_{\text {tor }}(\mathbb{Q})$ and $\operatorname{rank} E(\mathbb{Q}) \geq 1$. From the Lutz-Nagell theorem [10, p.240], $P$ cannot be of finite order. For more details on the extended results of pyramidal numbers and rank of elliptic curves, one can refer to [9].

\subsection{Convolution on the twisted Eisenstein series $S_{2 k+2, \chi_{i}}^{*} i=0,1$}

We prove the following result.

Lemma 3.3 Let $k \geq 1$. Then

$$
\begin{aligned}
S_{2 k+2, \chi_{0}}^{*}(\tau) & =4 \sum_{s=0}^{k-1}\left(\begin{array}{c}
2 k \\
2 s+1
\end{array}\right) S_{2 k-2 s, \chi_{1}}^{*}(\tau) S_{2 s+2, \chi_{1}}^{*}(\tau) \\
& =4 \sum_{s=0}^{k-1}\left(\begin{array}{c}
2 k \\
2 s+1
\end{array}\right) S_{2 k-2 s, \chi_{1}}(\tau) S_{2 s+2, \chi_{1}}(\tau)
\end{aligned}
$$

and

$$
D\left(S_{2 k, \chi_{0}}^{*}(\tau)\right)=2 \sum_{s=0}^{k-1}\left(\begin{array}{c}
2 k \\
2 s+1
\end{array}\right)\left(S_{2 k-2 s, \chi_{1}}^{*}(\tau)-S_{2 k-2 s, \chi_{0}}^{*}(\tau)\right) S_{2 s+2}^{*}(\tau) .
$$


Proof Let us put $N=2 L$ in (10). Then

$$
\begin{aligned}
\sum_{s=0}^{k-1}\left(\begin{array}{c}
2 k \\
2 s+1
\end{array}\right) \sum_{m=1}^{2 L-1} \sigma_{2 k-2 s-1}^{*}(m) \sigma_{2 s+1}^{*}(2 L-m) \\
=\sum_{s=0}^{k-1}\left(\begin{array}{c}
2 k \\
2 s+1
\end{array}\right)\left(\sum_{m=1}^{L} \sigma_{2 k-2 s-1}^{*}(2 m-1) \sigma_{2 s+1}^{*}(2 L-2 m+1)\right. \\
\left.\quad+\sum_{m=1}^{L-1} \sigma_{2 k-2 s-1}^{*}(2 m) \sigma_{2 s+1}^{*}(2 L-2 m)\right) \\
=\frac{1}{2}\left\{\sigma_{2 k+1}^{*}(2 L)-2 L \sigma_{2 k-1}^{*}(2 L)\right\} .
\end{aligned}
$$

From Theorem 2.2 and (14), we derive

$$
\begin{aligned}
& \sum_{s=0}^{k-1}\left(\begin{array}{c}
2 k \\
2 s+1
\end{array}\right)\left(\sum_{m=1}^{L} \sigma_{2 k-2 s-1}(2 m-1) \sigma_{2 s+1}(2 L-2 m+1)\right) \\
& \quad=\sum_{s=0}^{k-1}\left(\begin{array}{c}
2 k \\
2 s+1
\end{array}\right)\left(\sum_{m=1}^{L} \sigma_{2 k-2 s-1}^{*}(2 m-1) \sigma_{2 s+1}^{*}(2 L-2 m+1)\right) \\
& \quad=\frac{1}{2}\left\{2^{2 k+1} \sigma_{2 k+1}^{*}(L)-2 L \cdot 2^{2 k-1} \sigma_{2 k-1}^{*}(L)\right\}-\frac{1}{2} \cdot 2^{2 k}\left\{\sigma_{2 k+1}^{*}(L)-L \sigma_{2 k-1}^{*}(L)\right\} \\
& =\frac{1}{4} \sigma_{2 k+1}^{*}(2 L) .
\end{aligned}
$$

From (14) and (15), we find that

$$
\sum_{s=0}^{k-1}\left(\begin{array}{c}
2 k \\
2 s+1
\end{array}\right) \sum_{m=1}^{2 L-1}(-1)^{m+1} \sigma_{2 k-2 s-1}^{*}(m) \sigma_{2 s+1}^{*}(2 L-m)=\frac{1}{2}\left(2 L \cdot \sigma_{2 k-1}^{*}(2 L)\right) .
$$

From (15) and (16), we deduce our lemma.

We have the following theorem.

Theorem 3.4 Let $L, M, k$ be positive integers. Let

$$
U_{2 k}(M):=\sum_{s=0}^{k-1}\left(\begin{array}{c}
2 k \\
2 s+1
\end{array}\right) \sum_{m=0}^{M} \sigma_{2 k-2 s-1}(2 m-1) \sigma_{2 s+1}(2 M-(2 m-1)) .
$$

(a) If $(L, M)=1$, then

$$
U_{2 k}(L) U_{2 k}(M)=2^{2 k-1} U_{2 k}(L M) .
$$

(b) If $L=2^{e_{0}} p_{1}^{e_{1}} \cdots p_{r}^{e_{r}}$ with odd distinct primes $p_{i}$, then

$$
U_{2 k}(L)=2^{2 r-2+(2 k+1)\left(e_{0}-e_{1}-\cdots-e_{r}\right)} U_{2 k}\left(p_{1}^{e_{1}}\right) \cdots U_{2 k}\left(p_{r}^{e_{r}}\right) .
$$


Proof

(a) Since $(L, M)=1$, we can choose $2 \mid L$ and $2 \nmid M$. From the definition of $U_{2 k}(M)$ and (15), we obtain

$$
\begin{aligned}
U_{2 k}(L) U_{2 k}(M) & =\frac{1}{4} \sigma_{2 k+1}^{*}(2 L) \cdot \frac{1}{4} \sigma_{2 k+1}^{*}(2 M) \\
& =\frac{1}{4} \sigma_{2 k+1}^{*}(2 L) \cdot \frac{1}{4} \cdot 2^{2 k+1} \sigma_{2 k+1}^{*}(M) \\
& =2^{2 k-1}\left(\frac{1}{4} \sigma_{2 k+1}^{*}(2 L M)\right) \\
& =2^{2 k-1} U_{2 k}(L M) .
\end{aligned}
$$

(b) Since $L=2^{e_{0}} p_{1}^{e_{1}} \cdots p_{r}^{e_{r}}$ with odd distinct primes $p_{i}$, we have

$$
\begin{aligned}
U_{2 k}(L) & =\frac{1}{4} \sigma_{2 k+1}^{*}\left(2^{e_{0}} p_{1}^{e_{1}} \cdots p_{r}^{e_{r}}\right) \\
& =\frac{1}{4} \cdot 2^{(2 k+1) e_{0}} \sigma_{2 k+1}^{*}\left(p_{1}^{e_{1}} \cdots p_{r}^{e_{r}}\right) \\
& =\frac{1}{4} \cdot 2^{(2 k+1) e_{0}} \sigma_{2 k+1}^{*}\left(p_{1}^{e_{1}}\right) \cdots \sigma_{2 k+1}^{*}\left(p_{r}^{e_{r}}\right) \\
& =\frac{1}{4} \cdot 2^{(2 k+1) e_{0}} 2^{-(2 k+1) e_{1}} \sigma_{2 k+1}^{*}\left(2 p_{1}^{e_{1}}\right) \cdots 2^{-(2 k+1) e_{r}} \sigma_{2 k+1}^{*}\left(2 p_{r}^{e_{r}}\right) \\
& =\frac{1}{4} \cdot 2^{(2 k+1) e_{0}-(2 k+1) e_{1}-\cdots-(2 k+1) e_{r}}\left(\frac{1}{4} \sigma_{2 k+1}^{*}\left(2 p_{1}^{e_{1}}\right)\right) \cdots\left(\frac{1}{4} \sigma_{2 k+1}^{*}\left(2 p_{r}^{e_{r}}\right)\right) \cdot 4^{r} \\
& =2^{2 r-2+(2 k+1)\left(e_{0}-e_{1}-\cdots-e_{r}\right)} U_{2 k}\left(p_{1}^{e_{1}}\right) \cdots U_{2 k}\left(p_{r}^{e_{r}}\right)
\end{aligned}
$$

\subsection{Triangular and twisted triangular numbers: results and remarks}

(1) Using the theory of elliptic theta functions, Glaisher [11, p.300] considered Eq. (15).

(2) Four special cases for (15) and (16) are of interest here (see Table 6).

(3) In particular, if $L$ is an odd prime, then

$$
\frac{1}{2} \sum_{m=1}^{2 L-1}(-1)^{m+1} \sigma_{1}^{\prime \prime}(m) \sigma_{1}^{\prime \prime}(2 L-m)=\frac{L(L+1)}{2}=\sum_{k=1}^{L} k=T_{L}
$$

where $T_{L}$ is a triangular number. We note that

$$
\sum_{m=1}^{L-1}(-1)^{m+1} \sigma_{1}^{*}(m) \sigma_{1}^{*}(2 L-m)=\frac{\sigma_{1}^{*}(L)}{2}\left\{L+(-1)^{L} \sigma_{1}^{*}(L)\right\}
$$

\section{Table 6 Special cases of convolution sums}

\begin{tabular}{ll}
\hline Convolution sums & Convolution sums \\
\hline$\sum_{m=1}^{L} \sigma_{1}(2 m-1) \sigma_{1}(2 L-2 m+1)=\sigma_{3}^{*}(L)$ & $\sum_{m=1}^{L} \sigma_{1}(2 m-1) \sigma_{3}(2 L-2 m+1)=\sigma_{5}^{*}(L)$ \\
$\sum_{m=1}^{L L}(-1)^{m+1} \sigma_{1}^{*}(m) \sigma_{1}^{*}(2 L-m)=L \sigma_{1}^{*}(L)$ & $\sum_{m=1}^{2 L-1}(-1)^{m+1} \sigma_{1}^{*}(m) \sigma_{3}^{*}(2 L-m)=L \sigma_{3}^{*}(L)$ \\
\hline
\end{tabular}


by using $m \equiv 2 L-m(\bmod 2)$. If $L$ is odd, then

$$
\sum_{m=1}^{L-1}(-1)^{m} \sigma_{1}^{*}(m) \sigma_{1}^{*}(2 L-m)=\frac{\sigma_{1}^{*}(L)}{2}\left\{\sigma_{1}^{*}(L)-L\right\}
$$

(4) In particular, if $L=2 q-1$ is an odd prime, then

$$
\sum_{m=1}^{L-1}(-1)^{m} \sigma_{1}^{*}(m) \sigma_{1}^{*}(2 L-m)=\frac{L+1}{2}=\sum_{k=1}^{q} k^{0}=q .
$$

We introduce the twisted triangular numbers $T_{L}^{*}$ and $F_{L}^{*}$ given by

$$
T_{L}^{*}:=\frac{1}{2} \sum_{m=1}^{2 L-1}(-1)^{m+1} \sigma_{1}^{*}(m) \sigma_{1}^{*}(2 L-m), \quad F_{L}^{*}:=\sum_{m=1}^{2 L-2}(-1)^{m} \sigma_{1}^{*}(m) \sigma_{1}^{*}(4 L-2-m) .
$$

The first sixteen values of

$$
T_{L}, \quad T_{L}^{*}, \quad F_{L}:=\sum_{k=1}^{L} k^{0}, \quad F_{L}^{*}
$$

are given in Table 7.

We can see from Figure 3 and Table 7 that when $2 x-1$ (resp. $x$ ) is prime, the numbers

$$
F_{x}^{*}=\sum_{m=1}^{2 x-2}(-1)^{m} \sigma_{1}^{*}(m) \sigma_{1}^{*}(4 x-2-m)
$$

$\left(\right.$ resp. $\left.T_{x}^{*}:=\frac{1}{2} \sum_{m=1}^{2 x-1}(-1)^{m+1} \sigma_{1}^{*}(m) \sigma_{1}^{*}(2 x-m)\right)$ and $F_{x}=\sum_{k=1}^{x} k^{0}\left(\right.$ resp. $\left.T_{x}=\sum_{k=1}^{x} k\right)$ are the same.

(5) A similar question regarding such convolution formulas has been addressed previously in [12]: Can one find $r_{1}, r_{2}, s_{1}, s_{2}, m, \alpha_{1}, \beta_{1}, \beta$ in $\mathbb{Z}$ satisfying

$$
\sum_{k<\beta p / \beta_{1}} \sigma_{r_{1}, s_{1}}\left(\alpha_{1} k ; m\right) \sigma_{r_{2}, s_{2}}\left(\beta p-\beta_{1} k ; m\right)=\sum_{k=1}^{\frac{p-1}{2}} k^{u}
$$

\begin{tabular}{|c|c|c|c|c|c|c|c|c|c|c|c|c|c|c|c|}
\hline 2 & 3 & 4 & 5 & 6 & 7 & 8 & 9 & 10 & 11 & 12 & 13 & 14 & 15 & 16 & 17 \\
\hline 3 & 6 & 10 & 15 & 21 & 28 & 36 & 45 & 55 & 66 & 78 & 91 & 105 & 120 & 136 & 153 \\
\hline 2 & 6 & 8 & 15 & 24 & 28 & 32 & $\frac{117}{2}$ & 60 & 66 & 96 & 91 & 112 & 180 & 128 & 153 \\
\hline 2 & 3 & 4 & 5 & 6 & 7 & 8 & 9 & 10 & 11 & 12 & 13 & 14 & 15 & 16 & 17 \\
\hline 2 & 3 & 4 & 26 & 6 & 7 & 108 & 9 & 10 & 176 & 12 & 93 & 260 & 15 & 16 & 360 \\
\hline
\end{tabular}

for a fixed $u$ and for a fixed odd prime $p$ ?

We believe that such a problem is generally not easy to solve. Equations (17) and (18) are special cases for this question with $u=0$ and 1 . A similar result is presented in [12, (12)].

(6) From Tables 6 and 7, we can easily obtain values of $T_{L}^{*}$ for any prime $L$.

Table 7 Examples for $T_{L}^{*}, T_{L}, F_{L}$, and $F_{L}^{*}$ 

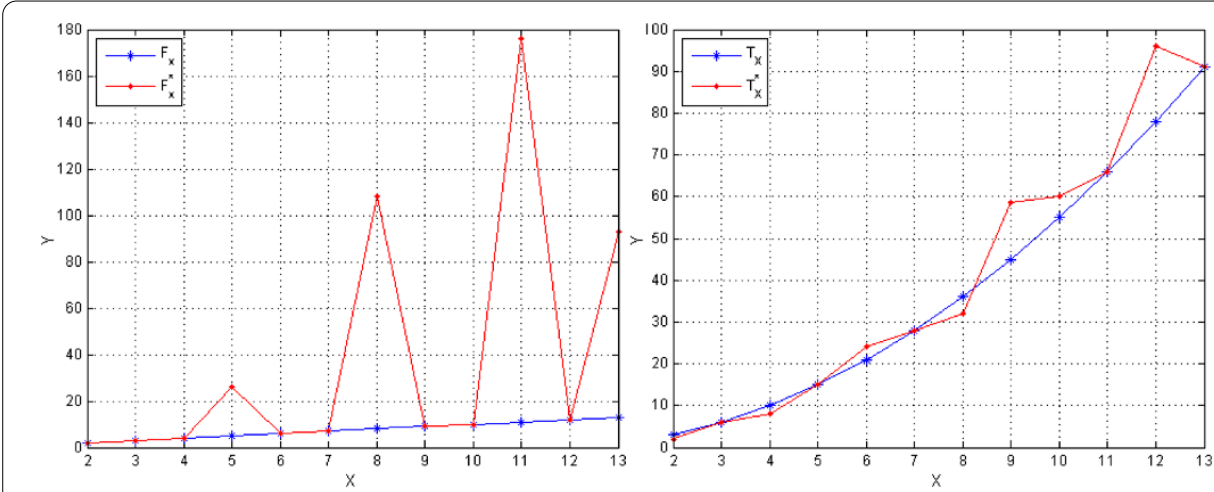

Figure $3 F_{L}, F_{L}^{*}, T_{L}^{*}$, and $T_{L}$.

Table 8 Triangular and twisted triangular numbers

\begin{tabular}{ll}
\hline Triangular number & Twisted triangular number \\
\hline$m, n:$ any positive integer & $m, n, \frac{m+n}{2}:$ odd prime numbers \\
$T_{m+n}=T_{m}+T_{n}+(m n)$ & $T_{m+n}^{*}=T_{m}^{*}+T_{n}^{*}+m n+\frac{m+n}{2}$ \\
{$[13,(2)]$} & \\
\hline$n:$ any positive integer & $n:$ odd prime number \\
$T_{2 n}-2 T_{n}=n^{2}$ & $T_{2 m_{n}}^{*}-4^{m} T_{n}^{*}=0$ \\
{$[14,(1.13)]$} & \\
\hline$n:$ any positive integer & $2 n-1, n-1:$ odd prime numbers \\
$T_{2 n-1}-2 T_{n-1}=n^{2}$ & $T_{2 n-1}^{*}-2 T_{n-1}^{*}=n^{2}$ \\
{$[14,(1.14)]$} & \\
\hline$n:$ any positive integer & $n, 5 n+2:$ odd prime numbers \\
$25 T_{n}+3=T_{5 n+2}$ & $25 T_{n}^{*}+3=T_{5 n+2}^{*}$ \\
{$[14,(4.2)]$} & \\
\hline
\end{tabular}

(7) In Table 8 we compare the properties of triangular numbers $T_{L}$ and twisted triangular numbers $T_{L}^{*}$.

Remark 3.5 (An application) Let us compute the quantity $\sum_{k=1}^{N-1} \sigma_{1}(k ; 4) \sigma_{1}(N-k ; 4)$, that is,

$$
\begin{aligned}
\sum_{k=1}^{N-1} \sigma_{1}^{*}(k ; 4) \sigma_{1}^{*}(N-k ; 4) & \\
= & \sum_{k=1}^{N-1}\left\{\sigma_{1}(k)-\sigma_{1}\left(\frac{k}{4}\right)\right\}\left\{\sigma_{1}(N-k)-\sigma_{1}\left(\frac{N-k}{4}\right)\right\} \\
= & \sum_{k=1}^{N-1} \sigma_{1}(k) \sigma_{1}(N-k)-2 \sum_{k<\frac{N}{4}} \sigma_{1}(N-4 k) \sigma_{1}(k) \\
& +\sum_{k<\frac{N}{4}} \sigma_{1}(k) \sigma_{1}\left(\frac{N}{4}-k\right) \\
= & \frac{1}{8}\left\{3 \sigma_{3}^{*}(N ; 4)-\sigma_{3}^{*}\left(\frac{N}{2}\right)-3 N \sigma_{1}^{*}(N ; 4)\right\},
\end{aligned}
$$


Table 9 Convolution sum when $N$ is prime

\begin{tabular}{lll}
\hline $\boldsymbol{N}$ : prime & $\sum_{\boldsymbol{k}=\mathbf{1}}^{N-1} \sigma_{\mathbf{1}}^{*}(\mathbf{k}) \boldsymbol{\sigma}_{\mathbf{1}}^{*}(\boldsymbol{N}-\boldsymbol{k})$ & $\sum_{\mathbf{k = 1}}^{\boldsymbol{N}-\mathbf{1}} \sigma_{\mathbf{1}}^{*}(\mathbf{k} ; \mathbf{4}) \boldsymbol{\sigma}_{\mathbf{1}}^{*}(\boldsymbol{N}-\mathbf{k} ; \mathbf{4})$ \\
\hline$N=2 q+1$ & $2 q^{2}(q+1)$ & $3 q^{2}(q+1)$ \\
\hline
\end{tabular}

where we refer to

$$
\sum_{k=1}^{N-1} \sigma_{1}(k) \sigma_{1}(N-k)=\frac{1}{12}\left\{5 \sigma_{3}(N)+(1-6 N) \sigma_{1}(N)\right\}
$$

in $[15,(3.10)]$ and

$$
\begin{aligned}
& \sum_{k<\frac{N}{4}} \sigma_{1}(k) \sigma_{1}(N-4 k) \\
& \quad=\frac{1}{48}\left\{\sigma_{3}(N)+(2-3 N) \sigma_{1}(N)+3 \sigma_{3}\left(\frac{N}{2}\right)+16 \sigma_{3}\left(\frac{N}{4}\right)+(2-12 N) \sigma_{1}\left(\frac{N}{4}\right)\right\}
\end{aligned}
$$

in [15, Theorem 4]. Combining (19) and (12), we have Table 9.

From Theorem 1.3, for $k=1$, we deduce that

$$
\begin{aligned}
& \sum_{k=1}^{N-1} \sigma_{1}^{*}(4 k ; 4) \sigma_{1}^{*}(4(N-k) ; 4) \\
& \quad=\frac{9}{80}\left\{\sigma_{3}^{*}(4 N ; 4)+\sigma_{3}^{*}(2 N)-16 N \sigma_{1}^{*}(4 N ; 4)+8 N \sigma_{1}^{*}(2 N)\right\} .
\end{aligned}
$$

Moreover, from (19) and (22), we see that

$$
\begin{aligned}
& \sum_{\substack{k=1 \\
4 \nmid k}}^{4 N-1} \sigma_{1}(k) \sigma_{1}(4 N-k)=\sum_{\substack{k=1 \\
4 \nmid k}}^{4 N-1} \sigma_{1}^{*}(k ; 4) \sigma_{1}^{*}(4 N-k ; 4) \\
& =\sum_{k=1}^{4 N-1} \sigma_{1}^{*}(k ; 4) \sigma_{1}^{*}(4 N-k ; 4)-\sum_{k=1}^{N-1} \sigma_{1}^{*}(4 k ; 4) \sigma_{1}^{*}(4 N-4 k ; 4) \\
& =\frac{1}{80}\left\{21 \sigma_{3}(4 N)-19 \sigma_{3}(2 N)-2 \sigma_{3}(N)+24 N \sigma_{1}(4 N)\right. \\
& \left.\quad-72 N \sigma_{1}(2 N)+48 N \sigma_{1}(N)\right\} .
\end{aligned}
$$

To refine the above formula, we use the following observation. Let $p$ be a prime and $k$, $N \in \mathbb{N}$. It is well known that

$$
\sigma_{k}(p N)-\left(p^{k}+1\right) \sigma_{k}(N)+p^{k} \sigma_{k}\left(\frac{N}{p}\right)=0
$$

from [2, p.26]. Now, if we set $p=2$ and use (23) and (24), we obtain

$$
\sum_{\substack{k=1 \\ 4 \nmid k}}^{4 N-1} \sigma_{1}(k) \sigma_{1}(4 N-k)=17 \sigma_{3}^{*}(N)=\sigma_{3}^{*}(2 N ; 4)+\sigma_{3}^{*}(2 N) .
$$


Table 10 Convolution sums and triangular numbers

\begin{tabular}{|c|c|c|}
\hline & Convolution sum & $t_{8}(N)$ \\
\hline$\overline{\sum_{m=1}^{4 N-1} \sigma_{1}(m) \sigma_{1}(4 N-m)}$ & $\frac{1}{12}\left\{5 \sigma_{3}(4 N)+(1-24 N) \sigma_{1}(4 N)\right\}$ & \\
\hline$\sum_{m=1}^{2 N} \sigma_{1}(2 m-1) \sigma_{1}(4 N-(2 m-1))$ & $\sigma_{3}^{*}(2 N)$ & $t_{8}(2 N-1)$ \\
\hline$\sum_{\substack{m=1 \\
4 \nmid m}}^{4 N-1} \sigma_{1}(m) \sigma_{1}(4 N-m)$ & $\sigma_{3}^{*}(2 N)+\sigma_{3}^{*}(2 N ; 4)$ & $17 t_{8}(N-1)$ \\
\hline$\sum_{\substack{m=1 \\
m \equiv 2 \bmod 4}}^{4 N-1} \sigma_{1}(m) \sigma_{1}(4 N-m)$ & $\sigma_{3}^{*}(2 N ; 4)$ & $9 t_{8}(N-1)$ \\
\hline
\end{tabular}

Remark 3.6 (Representations of $N$ as the sum of $m$ triangular numbers) We set $\Delta=$ $\left\{T_{k} \mid k=0,1,2, \ldots\right\}$. For $m \in \mathbb{N}$ and $N \in \mathbb{N} \cup\{0\}$, we let

$$
t_{m}(N)=\operatorname{card}\left\{\left(x_{1}, x_{2}, \ldots, x_{m}\right) \in \Delta \times \Delta \times \cdots \times \Delta \mid N=x_{1}+x_{2}+\cdots+x_{m}\right\}
$$

so that $t_{m}(N)$ counts the number of representations of $N$ as the sum of $m$ triangular numbers. For $N \in \mathbb{N} \cup\{0\}$, we have the classical result

$$
t_{8}(N)=\sigma_{3}(N+1)-\sigma_{3}\left(\frac{N+1}{2}\right)=\sigma_{3}^{*}(N+1) ;
$$

see $[16, \mathrm{p} .55]$.

From (26), (25), (20), Table 5, and [17, p.401], we obtain Table 10.

From Theorem 2.2, we derive

$$
t_{8}(2 N-1)=\sigma_{3}^{*}(2 N)=8 \sigma_{3}^{*}(N)=8 t_{8}(N-1), \quad t_{8}\left(2^{m}-1\right)=8^{m},
$$

and using Theorem 2.2 we get

$$
\begin{aligned}
t_{8}\left(2^{m} p_{1}^{e_{1}} \cdots p_{r}^{e_{r}}-1\right) & =\sigma_{3}^{*}\left(2^{m} p_{1}^{e_{1}} \cdots p_{r}^{e_{r}}\right)=8^{m} \sigma_{3}^{*}\left(p_{1}^{e_{1}}\right) \cdots \sigma_{3}^{*}\left(p_{r}^{e_{r}}\right) \\
& =8^{m} t_{8}\left(p_{1}^{e_{1}}-1\right) \cdots t_{8}\left(p_{r}^{e_{r}}-1\right)
\end{aligned}
$$

with $p_{i}$ odd distinct primes.

\subsection{Representation of an integer $\boldsymbol{N}$ as the sum of $s$ squares}

The $s$ squares problem is to count the number $r_{s}(N)$ of integer solutions $\left(x_{1}, \ldots, x_{s}\right)$ of the Diophantine equation

$$
x_{1}^{2}+\cdots+x_{s}^{2}=N
$$

where changing the sign of the order of the $x_{i}^{\prime} s$ gives distinct solutions. Explicit formulas for $r_{2}(N)$ were given by Legendre in 1798 and Gauss in 1801. In 1770 Lagrange gave formula for $r_{4}(N)$. Using the theory of theta functions, Jacobi obtained explicit formulas for $r_{s}(N)$, where $s=2,4,6,8$. Glaisher used elliptic function methods rather than modular functions to obtain ${ }_{16}(N)$. A formula for $r_{24}(n)$ was proved by Ramanujan. For more details, see [18] and references therein. In this subsection, we give a new formulation for $r_{8}(4 N)$ in terms of $\sigma_{3}^{*}$ and $\sigma_{3}$, and from our main result, we deduce a congruence formula for this number. Following Jacobi, we consider the equation

$$
\vartheta_{3}(0,-q)^{s}:=1+\sum_{N=1}^{\infty}(-1)^{N} r_{s}(N) q^{N},
$$


where $\vartheta_{3}(0, q)$ is the $z=0$ case of the theta function $\vartheta_{3}(z, q)$ in [19] given by

$$
\vartheta_{3}(0, q)=\sum_{j=-\infty}^{\infty} q^{j^{2}}
$$

Jacobi [20] proved

$$
\vartheta_{3}(0,-q)^{4}=1-8 \sum_{r=1}^{\infty}(-1)^{r-1} \frac{r q^{r}}{1+q^{r}}=1+8 \sum_{N=1}^{\infty}(-1)^{N}\left(\sum_{\substack{d \mid N, d>0 \\ 4 \nmid d}} d\right) q^{N} .
$$

From (29) and (23), we get a new formulation of $r_{8}(4 N)$ in terms of $\sigma_{3}^{*}(N)$ and $\sigma_{3}(N)$. In fact,

$$
\begin{aligned}
r_{8}(4 N)= & 16\left(\sigma_{1}(4 N)-4 \sigma_{1}(N)\right)+64 \sum_{\substack{k=1 \\
4 \nmid k}}^{4 N-1} \sigma_{1}(k) \sigma_{1}(4 N-k) \\
& +64 \sum_{k=1}^{N-1}\left\{\sigma_{1}(4 k)-4 \sigma_{1}(k)\right\}\left\{\sigma_{1}(4(N-k))-4 \sigma_{1}(N-k)\right\}
\end{aligned}
$$

and then

$$
r_{8}(4 N)=16\left\{56 \sigma_{3}^{*}(N)+15 \sigma_{3}(N)\right\},
$$

which gives the following theorem. Here,

$$
\begin{aligned}
& \sum_{k=1}^{N-1}\left\{\sigma_{1}(4 k)-4 \sigma_{1}(k)\right\}\left\{\sigma_{1}(4(N-k))-4 \sigma_{1}(N-k)\right\} \\
& \quad=\sum_{k=1}^{N-1}\left\{3 \sigma_{1}(k)-6 \sigma\left(\frac{k}{2}\right)\right\}\left\{3 \sigma_{1}(N-k)-6 \sigma\left(\frac{N-k}{2}\right)\right\} \\
& =9 \sum_{k=1}^{N-1} \sigma_{1}(k) \sigma_{1}(N-k)-36 \sum_{k<\frac{N}{2}} \sigma_{1}(N-2 k) \sigma_{1}(k)+36 \sum_{k<\frac{N}{2}} \sigma_{1}(k) \sigma_{1}\left(\frac{N}{2}-k\right) \\
& =\frac{3}{4}\left(\sigma_{3}(N)+4 \sigma_{3}\left(\frac{N}{2}\right)-\sigma_{1}(N)+2 \sigma_{1}\left(\frac{N}{2}\right)\right),
\end{aligned}
$$

we refer to $[15,(3.10),(4.4)]$.

From the above computation, we have a beautiful arithmetical results, which we give now.

Theorem 3.7 Let $N \in \mathbb{N}$. We have a formula

$$
r_{8}(4 N)=16\left\{56 \sigma_{3}^{* \prime}(N)+15 \sigma_{3}(N)\right\}
$$

and if $N \equiv-1(\bmod 8)$ is an odd integer, then

$$
r_{8}(4 N) \equiv 0 \quad(\bmod 9,088) .
$$


Proof By using $(30)$ and $\sigma_{3}(8 k-1) \equiv 0(\bmod 8)$, we get the theorem.

Remark 3.8 Let $M$ and $N$ be odd integers with $(M, N)=1$. From Theorem 3.7, we derive that

$$
\begin{aligned}
r_{8}(4 M N) & =1,136 \sigma_{3}(M) \sigma_{3}(N)=\frac{1}{1,136}\left(1,136 \sigma_{3}(M)\right)\left(1,136 \sigma_{3}(N)\right) \\
& =\frac{1}{1,136} r_{8}(4 M) r_{8}(4 N)
\end{aligned}
$$

and

$$
r_{8}\left(4 p_{1}^{e_{1}} \cdots p_{r}^{e_{r}}\right)=\left(\frac{1}{1,136}\right)^{r-1} r_{8}\left(4 p_{1}^{e_{1}}\right) \cdots r_{8}\left(4 p_{r}^{e_{r}}\right)
$$

Corollary 3.9 Let $N=p_{1}^{e_{1}} \cdots p_{r}^{e_{r}}$ be a prime decomposition of the integer $N$, and if $p_{i}^{e_{i}}=-1$ $(\bmod 8)$ for all $i=1, \ldots, r$, then we have

$$
r_{8}(4 N) \equiv 0 \quad\left(\bmod 2^{4+3 r} \cdot 71\right)
$$

4 The problem $\sigma_{1}^{*}(n)=\sigma_{1}^{*}(n+1)$

Sierpiński has asked if $\sigma_{1}(n)=\sigma_{1}(n+1)$ infinitely often. Jud McCranie found 832 solutions of

$$
\sigma_{1}(n)=\sigma_{1}(n+1) \quad \text { for } n<4.25 \times 10^{9} \text {; }
$$

see[21, p.103]).

We consider the values of $\sigma_{1}^{*}(n)=\sigma_{1}^{*}(n+1)$ and $\sigma_{1}^{*}(n)=\sigma_{1}^{*}(n+1)=\sigma_{1}^{*}(n+2)$.

\subsection{Further results}

First, we shall compare the above problem for $\sigma_{1}(n)$ and $\sigma_{1}^{*}(n)$.

The results of Table 11 were realized by combining several computers and by using Mathematica 8.0 software. It would be impossible for us to reproduce here all their details. It is clear, according to our numerical computations, that the study of the above problem with $\sigma_{1}^{*}$ is faster than that of the $\sigma_{1}$ one.

Table $11 \sigma_{1}^{*}(n)$ and $\sigma_{1}(n)$

\begin{tabular}{lll}
\hline $\boldsymbol{n}$ & $\boldsymbol{\sigma}_{\mathbf{1}}^{*}(\boldsymbol{n})=\boldsymbol{\sigma}_{\mathbf{1}}^{*}(\boldsymbol{n}+\mathbf{1})$ & $\boldsymbol{\sigma}_{\mathbf{1}}(\boldsymbol{n})=\boldsymbol{\sigma}_{\mathbf{1}}(\boldsymbol{n}+\mathbf{1})$ \\
\hline$n<200$ & $3,6,7,10,22,31,58$, & 14 \\
& $82,106,140,154$, & \\
& $160,166,180$ & \\
$n<4.25 \times 10^{9}$ & $\sharp\left\{n \mid \sigma_{1}^{*}(n)=\sigma_{1}^{*}(n+1)\right\}=1,870$ & $\sharp\left\{n \mid \sigma_{1}(n)=\sigma_{1}(n+1)\right\}=832$ \\
$n$ & $\sigma_{1}^{*}(n)=\sigma_{1}^{*}(n+1)=\sigma_{1}^{*}(n+2)$ & $\sigma_{1}(n)=\sigma_{1}(n+1)=\sigma_{1}(n+2)$ \\
$n<200$ & 6 & no \\
$n<4.25 \times 10^{9}$ & 6 & no \\
$n$ & $\sigma_{1}^{*}(n+2)=\sigma_{1}^{*}(n)+2$ & $\sigma_{1}(n+2)=\sigma_{1}(n)+2$ \\
twin prime & all & all \\
$m, n$ & $m \sigma_{1}^{*}(m)=n \sigma_{1}^{*}(n), m \neq n$ & $m \sigma_{1}(m)=n \sigma_{1}(n)$ \\
$1 \leq m, n \leq 7 \times 10^{4}$ & no & $m=12 q, n=14 q(q, 42)=1$ \\
\hline
\end{tabular}


Table 12

\begin{tabular}{llllll}
\hline & $\boldsymbol{\sigma}_{\mathbf{1}}(\boldsymbol{n}) \boldsymbol{= n}$ & $\boldsymbol{\sigma}_{\mathbf{1}}^{*}(\boldsymbol{n})=\boldsymbol{n}$ & $\boldsymbol{\sigma}_{\mathbf{1}}(\boldsymbol{n})=\boldsymbol{n}+\mathbf{2}$ & $\boldsymbol{\sigma}_{\mathbf{1}}^{*}(\boldsymbol{n})=\boldsymbol{n}+\mathbf{2}$ \\
\hline$n$ & 1 & $2^{m}, m \in \mathbb{N} \cup\{0\}$ & $n$ & none & $n=2 p$, with $p$ any odd prime \\
& $\sigma_{1}(n)=n+1$ & $\sigma_{1}^{*}(n)=n+1$ & & $\sigma_{1}(n)=n+3$ & $\sigma_{1}^{*}(n)=n+3$ \\
$n$ & prime & odd prime & $n$ & 4 & none \\
\hline
\end{tabular}

In fact, using Mathematica 8.0, we were able to obtain all positive integers for all $n<$ $4.25 \times 10^{9}$ in Table 11. Note that the results of $\sigma_{1}(n)$ in Table 11 are also presented in [21, p.100, pp.103-104].

\section{Theorem 4.1}

(a) If $2^{n}-1$ is an odd prime number, then $\sigma_{1}^{*}\left(2^{n}-1\right)=\sigma_{1}^{*}\left(2^{n}\right)$.

(b) If $p$ and $2 p+1$ are odd prime numbers, then $\sigma_{1}^{\prime \prime}(2 p)=\sigma_{1}^{\prime \prime}(2 p+1)$.

(c) If $2^{n}-1$ is an odd prime number, then we get $\sigma_{1}^{*}\left(2^{n}-2\right) \neq \sigma_{1}^{*}\left(2^{n}-1\right)=\sigma_{1}^{*}\left(2^{n}\right)$ with $n>3$ and $\sigma_{1}^{*}(6)=\sigma_{1}^{*}(7)=\sigma_{1}^{*}(8)$.

(d) If $n$ and $n+2$ are twin prime numbers, then $\sigma_{1}^{*}(n+2)=\sigma_{1}^{*}(n)+2$.

(e) If $n$ is a positive integer, then we get Table 12.

Proof Let $p$ be an odd positive integer. Using $\sigma_{1}(p)=p+1$ and $\sigma_{1}^{*}(2 m)=2 \sigma_{1}^{*}(m)$, we get (a), (b), (d), and (e). In (c), if $2^{n}-1$ is an odd prime number with $n>3$, then $n$ is an odd number. It is easy to verify that

$$
\sigma_{1}^{* \prime}\left(2^{n}-2\right)=2 \sigma_{1}^{*}\left(2^{n-1}-1\right)=2 \sigma_{1}^{*}\left(\left(2^{\frac{n-1}{2}}-1\right)\left(2^{\frac{n-1}{2}}+1\right)\right) \neq 2^{n}
$$

and $\sigma_{1}^{\prime \prime}\left(2^{n}-1\right)=\sigma_{1}^{\prime \prime}\left(2^{n}\right)=2^{n}$. It is clear that $\sigma_{1}^{\prime \prime}(6)=\sigma_{1}^{\prime \prime}(7)=\sigma_{1}^{\prime \prime}(8)$.

Corollary 4.2 Let $p$ be an odd prime. If $L=2^{n}-1$ is an odd prime or $L=2 p$, and $2 p+1$ is prime, then

$$
\begin{aligned}
\sigma_{1}(2 L+1)= & \frac{1}{2}\left\{\sigma_{1}(2 L-1)+5 \sigma_{1}^{*}(L)\right. \\
& \left.+\sum_{m=1}^{2 L-2}(-1)^{m+1} \sigma_{1}^{*}(m+1)\left(\sigma_{1}^{*}(2 L+1-m)-\sigma_{1}^{*}(2 L-m-1)\right)\right\} .
\end{aligned}
$$

Proof From Table 5, we get

$$
\begin{aligned}
& (L+1) \sigma_{1}^{*}(L+1)-L \sigma_{1}^{*}(L) \\
& =\sum_{m=1}^{2 L+1}(-1)^{m+1} \sigma_{1}^{*}(m) \sigma_{1}^{*}(2 L+2-m)-\sum_{m=1}^{2 L-1}(-1)^{m+1} \sigma_{1}^{*}(m) \sigma_{1}^{*}(2 L-m) \\
& =\sum_{m=1}^{2 L-1}(-1)^{m+1} \sigma_{1}^{*}(m)\left\{\sigma_{1}^{*}(2 L+2-m)-\sigma_{1}^{*}(2 L-m)\right\}-\sigma_{1}^{*}(2 L) \sigma_{1}^{*}(2)+\sigma_{1}^{*}(2 L+1) \\
& =\sigma_{1}^{*}(1)\left\{\sigma_{1}^{*}(2 L+1)-\sigma_{1}^{*}(2 L-1)\right\}+\sum_{m=2}^{2 L-1}(-1)^{m+1} \sigma_{1}^{*}(m)\left\{\sigma_{1}^{*}(2 L+2-m)-\sigma_{1}^{*}(2 L-m)\right\} \\
& -4 \sigma_{1}^{*}(L)+\sigma_{1}^{*}(2 L+1) .
\end{aligned}
$$


From Theorem 4.1(a), (b), we know that $\sigma_{1}^{*}(L+1)=\sigma_{1}^{*}(L)$ and $\sigma_{1}^{*}(2 L+1)=\sigma_{1}(2 L+1)$; hence, the proof is completed.

Lemma 4.3 Let $I_{i}:=\left\{n \in \mathbb{N} \mid \sigma_{1}^{*}(n)=\sigma_{1}^{*}(n+i)\right\}$ with $i \in \mathbb{N}$. Then $\delta_{i, 2^{m_{i}}}: I_{i} \rightarrow I_{2^{m_{i}}}, \delta_{i, 2^{m_{i}}}(n)=$ $2^{m} n$, is an injective map. In particular, there exists an injective sequence of $I_{2}$,

$$
I_{1} \stackrel{\delta_{1,2}}{\longrightarrow} I_{2} \stackrel{\delta_{2,4}}{\longrightarrow} I_{4} \stackrel{\delta_{4,8}}{\longrightarrow} I_{8} \rightarrow \cdots
$$

satisfying $\delta_{2^{m}, 2^{m+1}}$ injective map.

Proof Let $n \in I_{i}$. By the definition of $I_{i}$, we deduce that $\sigma_{1}^{*}(n)=\sigma_{1}^{*}(n+i)$. From Theorem 2.2, we get $\sigma_{1}^{*}\left(2^{m} n\right)=\sigma_{1}^{*}\left(2^{m} n+2^{m} i\right)$ and $2^{m} n \in I_{2^{m}}$. And let $n \in I_{i}$ and $l \in I_{i}$ with $n \neq l$, then $2^{m} n \neq 2^{m} l$ and $\sigma_{1}^{*}\left(2^{m} n\right)=\sigma_{1}^{*}\left(2^{m} n+i\right), \sigma_{1}^{*}\left(2^{m} l\right)=\sigma_{1}^{*}\left(2^{m} l+i\right)$. Therefore, $\delta_{i, 2^{m}}$ is an injective map. It is clear that $\delta_{2^{m}, 2^{m+1}}$ is an injective map.

By Lemma 4.3, we deduce the corollary.

Corollary 4.4 Let $m$ be a nonegative integer. If $\sharp\left\{n \mid \sigma_{1}^{* \prime}(n)=\sigma_{1}^{* \prime}(n+1)\right\}>\infty$, then

$$
\sharp\left\{n \mid \sigma_{1}^{*}(n)=\sigma_{1}^{*}\left(n+2^{m}\right)\right\}>\infty .
$$

\subsection{Remarks and examples}

We list the following interesting remarks and examples.

\section{Remark 4.5}

1. (Sophie Germain primes) Note that if there are an infinite number of Sophie Germain primes, then the problem $\sigma_{1}^{*}(n)=\sigma_{1}^{*}(n+1)$ has an infinite number of solutions (in terms of prime numbers).

2. (Mersenne numbers) Assume that $\sigma_{1}^{\prime \prime}(m)=\sigma_{1}^{\prime \prime}(m+1)$. Then, by Theorem 2.2, we have $\sigma_{1}^{*}\left(2^{l} m\right)=\sigma_{1}^{*}\left(2^{l} m+2^{l}\right)$. If the cardinal of the Mersenne primes or the cardinal of primes $p$, such that $2 p+1$ is also prime, is infinite, then by using Theorem 4.1(a), (b), the number of $m$ satisfying $\sigma_{1}^{\prime \prime}(m)=\sigma_{1}^{\prime \prime}(m+1)$ and $\sigma_{1}^{\prime \prime}(m)=\sigma_{1}^{\prime \prime}\left(m+2^{l}\right)$ is infinite.

3. (Perfect numbers) A positive integer $n$ is called perfect if $\sigma_{1}(n)=2 n$. Euclid and Euler showed that all even perfect numbers are of the form

$$
2^{p-1}\left(2^{p}-1\right) \quad \text { such that } 2^{p}-1 \text { is a Mersenne prime. }
$$

In our case, we observe that $\sigma_{1}^{*}\left(2^{p-1}\left(2^{p}-1\right)\right) \neq 2 \cdot 2^{p-1}\left(2^{p}-1\right)$. If there exists an odd positive integer $m$ satisfying $\sigma_{1}(m)=2 m$, then the number of $n$ satisfying $\sigma_{1}^{*}(n)=2 n$ is infinite, that is, $\sigma_{1}^{*}\left(2^{l} m\right)=2^{l} \sigma_{1}^{*}(m)=2\left(2^{l} m\right)$.

\subsection{Numerical computations for the truncated sets $I_{i}(N)$}

Let $N$ be a positive integer and set $I_{i}(N)=I_{i} \cap\{1,2, \ldots, N\}$. From Lemma 4.3 we see that the restricted map $\delta_{i, 2^{m}}(n)=2^{m} n$ is still injective between $I_{i}(N)$ and $I_{2^{m}}\left(2^{m} N\right)$.

In this section, using Mathematica 8.0 we compute the sets $I_{i}(N)$ for $N=100,000=$ $2^{5} \cdot 5^{5}$ and $i=2,4,8,16,32$. We obtain the following lists. 
(a) $I_{2}(100,000)=\{6,12,14,20,33,44,62,92,116,138,164,212,254,280,308,320$, $332,356,452,524,572,692,716,764,932,956,1,004,1,124,1,172,1,436,1,496$, 1,562, 1,676, 1,724, 1,772, 1,964, 2,002, 2,036, 2,132, 2,372, 2,564, 2,598, 2,612, 2,636, 2,732, 2,876, 2,913, 2,972, 3,044, 3,228, 3,236, 3,344, 3,408, 3,644, 3,812, 4,052, 4,076, 4,124, 4,187, 4,196, 4,292, 4,412, 4,728, 4,892, 4,916, 5,156, 5,170, 5,636, 5,756, 5,804, 5,924, 5,996, 6,044, 6,236, 6,332, 6,404, 6,764, 6,932, 7,169, $7,244,7,424,7,556,7,604,7,724,7,892,8,012,8,050,8,156,8,234,8,252,8,276$, 8,516, 8,564, 8,930, 9,092, 9,356, 9,359, 9,404, 9,572, 9,596, 9,836, 10,172, 10,196, $10,772,10,796,10,964,11,012,11,276,11,612,11,756,11,852,11,876,12,092,12,212$, $12,565,13,196,13,316,13,436,13,556,13,652,13,796,13,964,14,156,14,372,14,492$, $15,044,15,085,15,116,15,212,15,284,15,404,15,452,15,644,16,076,16,120,16,292$, $16,376,16,382,16,844,17,084,17,396,17,492,17,564,17,636,17,924,18,170,18,932$, $19,172,19,484,19,676,19,772,20,012,20,156,20,204,20,324,20,684,20,924$, 21,116, 21,212, 21,332, 21,461, 21,518, 21,596, 21,764, 22,004, 22,556, 22,844, 22,964, 23,396, 23,612, 24,212, 24,404, 24,452, 24,524, 24,692, 24,881, 25,019, 25,052, 25,076, 25,292, 25,316, 25,338, 25,796, 25,964, 26,084, 26,204, 26,252, 26,324, $26,609,27,044,27,596,27,932,28,172,28,316,28,412,28,484,28,604,28,772$, 28,844, 29,396, 29,696, 29,732, 30,164, 30,572, 30,596, 30,764, 31,292, 31,364, $31,532,31,604,32,276,32,372,32,444,32,804,32,972,33,092,33,590,34,052$, $34,652,34,772,34,964,35,358,35,804,35,876,36,116,36,236,36,884,37,172$, $37,484,37,676,37,892,37,916,38,156,38,516,38,756,39,164,40,244,40,364$, 40,458, 40,652, 41,012, 41,084, 41,252, 41,324, 42,116, 42,356, 42,452, 42,764, $42,836,42,932,43,124,43,196,43,532,44,684,45,078,45,284,45,476,45,572$, $45,884,46,076,46,196,46,316,46,796,46,863,47,132,47,204,47,252,47,324$, 47,636, 47,756, 47,792, 47,978, 48,044, 48,164, 48,404, 48,476, 48,812, 49,052, $49,225,49,316,50,612,50,684,51,164,51,284,51,596,51,692,51,835,51,836$, $52,004,52,196,52,916,53,058,53,252,53,804,53,852,53,963,54,212,54,476$, 54,596, 55,052, 55,532, 55,604, 55,652, 55,748, 56,036, 56,324, 56,612, 56,636, 56,996, 57,212, 57,284, 57,956, 58,056, 58,244, 58,484, 58,676, 58,796, 58,964, $59,132,59,324,59,516,59,756,59,987,60,404,60,644,60,692,60,932,61,076$, 61,604, 61,832, 62,276, 62,516, 63,092, 63,164, 63,212, 63,692, 64,004, 64,364, 65,012, 65,204, 65,684, 65,972, 66,212, 66,692, 67,244, 67,292, 67,532, 67,724, 68,636, 68,732, 69,164, 69,332, 69,404, 70,316, 70,676, 70,724, 71,756, 71,924, 72,164, 72,524, 72,596, 72,764, 72,836, 72,932, 73,364, 73,772, 73,844, 74,924, 75,092, 75,108, 75,212, 75,596, 76,652, 77,057, 77,204, 77,276, 77,492, 77,564, 77,732, 78,212, 78,236, 78,644, 78,836, 79,004, 79,292, 79,556, 79,652, 79,676, $79,964,80,252,80,996,81,055,81,476,81,572,81,644,81,764,82,772,83,012$, $83,036,83,084,83,156,83,516,83,684,83,756,83,852,84,044,84,356,84,596$, $84,716,84,884,85,364,85,532,85,676,86,170,86,444,86,804,86,852,87,212$, $87,572,88,052,88,316,88,532,89,036,89,084,89,372,89,396,89,636,89,732$, $89,876,89,924,90,164,91,004,91,412,91,772,92,396,93,116,93,284,93,356$, 93,836, 94,244, 94,412, 94,676, 95,012, 95,276, 95,636, 95,924, 96,812, 96,956, 97,124, 97,892, 98,036, 98,204, 98,444, 98,732, 98,996, 99,638, 99,884 \}.

(b) $I_{4}(100,000)=\{12,24,28,40,51,66,88,115,124,184,232,276,319,328,424,508$, $560,616,640,664,712,904,1,003,1,048,1,144,1,384,1,432,1,528,1,864,1,912$, 2,008, 2,248, 2,344, 2,585, 2,872, 2,992, 3,124, 3,352, 3,448, 3,544, 3,928, 4,004, 
4,072, 4,183, 4,195, 4,264, 4,744, 5,128, 5,196, 5,224, 5,272, 5,464, 5,752, 5,826, $5,944,5,959,6,088,6,456,6,472,6,688,6,816,7,288,7,624,8,104,8,152,8,248$, $8,374,8,392,8,584,8,824,9,456,9,784,9,832,10,312,10,340,11,272,11,512,11,608$, $11,659,11,848,11,992,12,088,12,367,12,472,12,561,12,664,12,808,13,528,13,581$, $13,864,14,338,14,488,14,848,15,112,15,208,15,365,15,448,15,784,16,024,16,100$, $16,312,16,468,16,504,16,552,17,032,17,128,17,860,18,184,18,712,18,718,18,808$, $19,144,19,192,19,672,20,344,20,392,20,541,21,544,21,592,21,928,22,024,22,552$, $23,224,23,512,23,704,23,752,24,184,24,424,25,130,26,392,26,632,26,872$, 27,112, 27,304, 27,592, 27,928, 28,312, 28,744, 28,984, 29,393, 30,088, 30,170, $30,232,30,424,30,568,30,808,30,904,31,288,32,152,32,240,32,584,32,665$, $32,752,32,764,33,688,34,168,34,792,34,984,35,128,35,272,35,848,36,340$, $37,864,38,344,38,968,39,352,39,544,39,913,40,024,40,312,40,408,40,648$, 41,368, 41,848, 42,232, 42,423, 42,424, 42,664, 42,922, 43,036, 43,192, 43,528, 44,008, 45,112, 45,688, 45,928, 46,792, 47,224, 47,841, 48,424, 48,808, 48,904, $49,048,49,384,49,762,50,038,50,104,50,152,50,435,50,584,50,632,50,676$, $51,592,51,928,52,168,52,408,52,504,52,648,53,218,54,088,55,192,55,864$, $56,344,56,632,56,824,56,968,57,208,57,544,57,688,58,792,59,392,59,464$, 60,328, 61,144, 61,192, 61,528, 62,584, 62,728, 63,064, 63,208, 64,552, 64,744, 64,888, 65,608, 65,944, 66,184, 67,180, 68,104, 69,304, 69,544, 69,928, 70,716, $71,608,71,752,72,232,72,472,73,768,74,344,74,968,75,352,75,784,75,832$, 76,312, 77,032, 77,512, 78,328, 80,488, 80,728, 80,916, 81,304, 82,024, 82,168, $82,504,82,648,84,232,84,712,84,904,85,528,85,672,85,864,86,248,86,392$, 87,064, 88,303, 89,368, 90,156, 90,568, 90,952, 91,144, 91,768, 92,152, 92,392, 92,599, 92,632, 93,592, 93,726, 94,264, 94,408, 94,504, 94,648, 95,272, 95,512, 95,584, 95,956, 96,088, 96,328, 96,808, 96,952, 97,624, 98,104, 98,450, 98,632\}.

(c) $I_{8}(100,000)=\{15,24,48,56,69,80,87,102,132,175,176,230,248,368,464,552$, $638,656,689,848,1,016,1,120,1,127,1,232,1,280,1,328,1,349,1,424,1,808,2,006$, 2,096, 2,288, 2,768, 2,864, 3,056, 3,728, 3,824, 4,016, 4,496, 4,688, 5,170, 5,744, 5,984, 6,248, 6,704, 6,896, 7,088, 7,856, 8,008, 8,144, 8,366, 8,390, 8,528, 9,488, $10,256,10,392,10,448,10,544,10,928,11,504,11,652,11,888,11,918,12,176,12,912$, $12,944,13,376,13,632,14,576,15,248,16,208,16,304,16,496,16,748,16,784,17,168$, $17,648,18,912,19,511,19,568,19,664,19,829,20,624,20,680,22,544,23,024$, 23,216, 23,318, 23,696, 23,984, 24,176, 24,597, 24,734, 24,944, 25,122, 25,328, 25,616, 27,056, 27,162, 27,728, 28,676, 28,976, 29,696, 30,224, 30,416, 30,730, $30,896,31,568,32,048,32,200,32,624,32,936,33,008,33,104,34,064,34,256$, $35,720,36,368,37,391,37,424,37,436,37,616,37,901,38,288,38,384,39,344$, 40,688, 40,784, 41,082, 43,088, 43,184, 43,856, 44,048, 45,104, 45,925, 46,448, 47,024, 47,408, 47,487, 47,504, 48,368, 48,848, 50,260, 52,784, 53,264, 53,744, $54,224,54,608,55,184,55,856,56,624,57,488,57,968,58,786,59,125,60,176$, $60,340,60,464,60,848,61,053,61,136,61,616,61,808,62,576,64,304,64,480$, 65,168, 65,330, 65,504, 65,528, 67,376, 68,336, 69,584, 69,968, 70,256, 70,544, 71,696, 72,680, 75,728, 76,688, 77,936, 78,704, 79,088, 79,826, 80,048, 80,624, $80,816,81,296,81,989,82,736,83,696,84,464,84,846,84,848,85,328,85,844$, $86,072,86,384,87,056,88,016,90,224,91,376,91,856,93,584,94,448,95,682$, $96,848,97,557,97,616,97,808,98,096,98,768,99,524,99,827\}$. 
(d) $I_{16}(100,000)=\{30,48,55,96,112,138,160,174,204,205,264,350,352,355,460$, 496, 736, 928, 1,104, 1,276, 1,293, 1,312, 1,378, 1,696, 2,032, 2,240, 2,254, 2,464, 2,560, 2,656, 2,698, 2,848, 3,277, 3,616, 3,669, 4,012, 4,192, 4,576, 5,536, 5,728, 6,112, 7,456, 7,648, 8,032, 8,992, 9,376, 9,853, 10,340, 11,488, 11,968, 12,496, 12,549, $13,408,13,792,13,899,14,176,14,857,15,712,16,016,16,288,16,732,16,780,17,056$, $18,976,20,512,20,784,20,896,21,088,21,856,23,008,23,287,23,304,23,776$, $23,836,24,352,25,824,25,888,26,077,26,752,27,264,29,152,30,496,32,416$, $32,608,32,992$, 33,496, 33,568, 34,336, 35,296, 36,669, 37,824, 39,022, 39,136, $39,187,39,328,39,658,41,248,41,360,41,525,45,088,46,048,46,432,46,636$, 47,392, 47,968, 48,352, 49,194, 49,468, 49,888, 50,244, 50,656, 51,232, 54,112, $54,324,55,456,56,743,57,352,57,952,59,392,60,448,60,832,61,460,61,792$, 63,136, 64,096, 64,400, 64,963, 65,248, 65,872, 66,016, 66,208, 68,128, 68,512, $71,440,72,736,73,321,74,782,74,848,74,872,75,232,75,802,76,576,76,768$, $78,688,81,376,81,568,82,164,85,839,86,176,86,368,87,712,88,096,90,208$, 90,637, 91,850, 92,896, 94,048, 94,816, 94,974, 95,008, 95,113, 96,193, 96,736, $97,696\}$.

(e) $I_{32}(100,000)=\{60,96,110,177,192,224,276,303,320,348,408,410,528,605$, 700, 704, 710, 749, 920, 992, 1,045, 1,157, 1,472, 1,856, 2,208, 2,552, 2,567, 2,586, 2,624, 2,756, 3,392, 4,064, 4,480, 4,508, 4,533, 4,928, 5,120, 5,312, 5,396, 5,696, 6,554, 7,232, 7,338, 7,697, 8,024, 8,384, 9,152, 10,547, 11,072, 11,456, 12,224, 13,199, $14,912,15,296,16,064,16,345,17,984,18,752,19,706,20,381,20,680,21,197,21,797$, 22,976, 23,936, 24,992, 25,075, 25,098, 26,816, 27,584, 27,798, 28,352, 29,321, $29,365,29,714,31,424,32,032,32,576,32,849,33,464,33,560,34,112,37,929$, $37,952,41,024,41,568,41,792,42,176,43,712$, 46,016, 46,574, 46,608, 47,552, $47,672,48,704,51,648,51,776,52,154,53,504,54,528,58,304,60,992,61,705$, $64,832,65,216,65,984,66,992,67,136,68,672,70,049,70,592,73,338,75,648$, $78,044,78,272,78,374,78,656,79,316,82,496,82,720,83,050,85,769,89,033$, 90,176, 92,096, 92,864, 93,272, 94,784, 95,936, 96,704, 98,388, 98,936, 99,776\}.

We conclude this paper by the following remark.

Remark 4.6 According to the obtained numerical results, it is interesting to study lower and upper bounds for the cardinality of the sets $I_{i}(N)$. In the same manner we can also study the asymptotic behavior of this cardinality. This would allow us to know whether or not the full $I_{i}$ is infinite.

Competing interests

The authors declare that they have no competing interests.

Authors' contributions

All authors read and approved the final manuscript.

\section{Author details}

${ }^{1}$ National Institute for Mathematical Sciences, Yuseong-daero 1689-gil, Yuseong-gu, Daejeon, 305-811, South Korea. ${ }^{2}$ Département de mathématiques, Université d'Evry Val d’Essonne, Bâtiment I.B.G.B.I., 3ème étage, 23 Boulevard de France, 91037 Evry cedex, France.

\section{Acknowledgements}

Dedicated to Professor Hari M Srivastava.

This research was supported by the National Institute for Mathematical Sciences (NIMS) grant funded by the Korea government (B21303) and the 'Equipe Ananlyse et Probabilités' of the Department of Mathematics at University of Evry. 


\section{References}

1. Berndt, BC: Ramanujan's Notebook, Part II. Springer, Berlin (1989)

2. Williams, KS: Number Theory in the Spirit of Liouville. London Mathematical Society Student Texts, vol. 76. Cambridge University Press, Cambridge, (2011)

3. Royer, E: Evaluating convolution sums of the divisor function by quasimodular forms. Int. J. Number Theory 3(2), 231-261 (2007)

4. Ramakrishnan, B, Sahu, B: Evaluation of the convolution sums $\sum_{\mid+15 m=n} \sigma(l) \sigma(m)$ and $\sum_{3 /+5 m=n} \sigma(l) \sigma(m)$ and an application. Int. J. Number Theory (2012, accepted)

5. Alaca, S, Williams, KS: Evaluation of the convolution sums $\sum_{1+6 m=n} \sigma(l) \sigma(m)$ and $\sum_{21+3 m=n} \sigma(l) \sigma(m)$. J. Number Theory 124(2), 491-510 (2007)

6. Alaca, A, Alaca, S, Uygul, F, Williams, KS: Restricted Eisenstein series and certain convolution sums. J. Comb. Number Theory 3, 1-14 (2011)

7. Adiga, C, Ramaswamy, HN: A note on certain divisibility problem. Int. J. Math. Anal. 2(24), 1157-1161 (2008)

8. Simsek, Y: Elliptic analogue of the Hardy sums related to elliptic Bernoulli functions. Gen. Math. 15(2-3), 3-23 (2007)

9. Gyory, K, Dujella, A, Pinter, A: On the power values of pyramidal numbers, I. Acta Arith. 155, 217-226 (2012)

10. Silverman, JH: The Arithmetic of Elliptic Curves. Springer, Berlin (1986)

11. Dickson, LE: History of the Theory of Numbers, vol. II. Chelsea, New York (1952)

12. Kim, A, Kim, D, Li, Y: Convolution sums arising from the divisor functions. J. Korean Math. Soc. 50(2), $331-360$ (2013)

13. Garge, AS, Shirali, SA: Triangular numbers. Resonance 17(7), 672-681 (2012)

14. Hoggatt, VE Jr., Bicknell, M: Triangular numbers. Fibonacci Q. 12, 221-230 (1974)

15. Huard, JG, Ou, ZM, Spearman, BK, Williams, KS: Elementary evaluation of certain convolution sums involving divisor functions. In: Number Theory for the Millennium, vol. II, pp. 229-274 (2002)

16. Cheng, N, Williams, KS: Evaluation of some convolution sums involving the sum of divisors functions. Yokohama Math. J. 52, 39-57 (2005)

17. Andrew, GE, Berndt, BC: Ramanujan's Lost Notebook, Part I. Springer, Berlin (2005)

18. Milne, S: New infinite families of exact sums of squares formulas, Jacobi elliptic functions, and Ramanujan's tau function. Proc. Natl. Acad. Sci. USA 93, 15004-15008 (1996)

19. Whittaker, ET, Watson, GN: A Course of Modern Analysis, 4th edn., pp. 464-498. Cambridge University Press, Cambridge (1927)

20. Jacobi, CGJ: Fundamenta Nova Theoriae Functionum Ellipticarum, Sumptibus Fratrum Bornträger. Reprinted in C.G.J. Jacobi, Gesammelte Werke, vol. 1, pp. 49-239. Reimer, Berlin (1881-1891)

21. Guy, RK: Unsolved Problems in Number Theory. Springer, Berlin (2004)

doi:10.1186/1687-1812-2013-81

Cite this article as: Kim and Bayad: Convolution identities for twisted Eisenstein series and twisted divisor functions. Fixed Point Theory and Applications 2013 2013:81

\section{Submit your manuscript to a SpringerOpen ${ }^{\circ}$ journal and benefit from:}

- Convenient online submission

- Rigorous peer review

- Immediate publication on acceptance

- Open access: articles freely available online

- High visibility within the field

- Retaining the copyright to your article 